\title{
Der Intensivpatient hat Besuch - haben wir nicht schon genug zu tun mit seiner Behandlung?
}

\author{
Gerhard Jorch
}

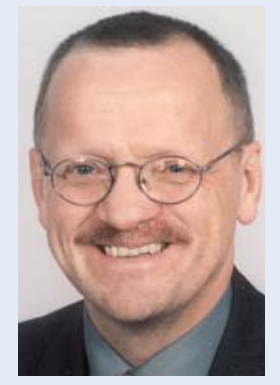

Gerhard Jorch
Korrespondenzadresse Prof. Dr. Gerhard Jorch Universitätsklinikum Magdeburg

Universitätskinderklinik Leipziger Str. 44 39112 Magdeburg gerhard.jorch@med. ovgu.de
D er Autor dieses Editorials ist Kinderarzt. Kinderärzte müssen Gefühle für Kinder haben. Noch wichtiger ist es aber, dass sie „Elternversteher" sind, da diese ohnehin Verhandlungspartner des Kinderarztes sind und weniger das diesbezüglich noch eingeschränkt „mündige“ Kind. Es ist seit Jahrzehnten üblich, Eltern auf neonatologischen Intensivstationen rund um die Uhr Zugang zu gewähren und sie in die Betreuung ihres Kindes mit einzubeziehen.

Zugegebenermaßen ist die Situation bei erwachsenen Patienten bereits bei der Einstiegsentscheidung schwieriger: Will der Patient überhaupt, dass Angehörige seine Interessen vertreten? Welche Person(en) aus seinem Umfeld sollen welche Entscheidungen für ihn treffen, durch welchen Angehörigen fühlt er sich am besten beraten? Wessen Besuch empfindet er als Hilfe und wen möchte er eigentlich gar nicht sehen an seinem Bett? Seine Rolle ist völlig anders als im sonstigen Leben. Sein soziales Beziehungsgeflecht muss in dieser Rolle möglicherweise neu definiert werden. Eher selten hat er vorher und schon gar nicht schriftlich festgelegt, wem das Fachpersonal Zugang gewähren soll. Selbst wenn, ist diese Festlegung schon deshalb unsicher, weil sich die meisten Menschen im Vorhinein die konkrete Situation, in der sie dann sind, nicht vorstellen können.

In dieser Ausgabe von Intensivup2date befassen sich Bone et al. mit dem Thema „Angehörige auf der Intensivstation“. Sie stellen Fakten aus Sicht des Patienten zusammen. So zeigen Studien, dass Patientenzufriedenheit und Outcome mit dem „Angehörigenmanagement" korrelieren. In einer australischen Studie wird von den Angehörigen insbesondere die Gesprächsbereitschaft, das Mitgefühl und der Respekt vom Pflegepersonal gewürdigt - auch bei Patienten, die am Ende verstarben. In der einzigen deutsch-sprachigen Studie entstand Unzufriedenheit vor allem durch zwei Faktoren: Erstens unzureichen-der Pflegepersonalschlüssel (plausibel), zweitens schriftlich hinterlegte Aufnahmeund Verlegungskriterien (?).
Übrigens wird eine gute Kommunikation zwischen Fachpersonal und Angehörigen auch vom Fachpersonal als stressmindernd empfunden. Für die Fähigkeit des letzteren zur Selbstkritik spricht, dass die Qualität der Kommunikation vom Fachpersonal kritischer als von den Angehörigen selbst gesehen wurde. Ein weiterer wertvoller Hinweis aus dem Artikel ist, dass die auskunftsgebende Person nicht zu häufig wechseln, ihre Auskünfte mit dem übrigen Fachpersonal absprechen und im Gespräch mit den Angehörigen ihren Redeanteil nicht zu hoch ansetzen, sondern Zeit für das Zuhören lassen sollte.

Das Gesagte muss unbedingt kurz in der Patientenakte dokumentiert werden, so dass in einer unübersichtlichen Notsituation den Angehörigen nicht plötzlich etwas anderes mitteilt wird. Dadurch würden die Angehörigen jegliches Vertrauen verlieren.

Zuletzt weisen die Autoren darauf hin, dass auch unzureichende räumliche Verhältnisse einer Intensivstation das Bemühen um Angehörigenzufriedenheit erschweren. Da die Planung und der Bau von Intensivstationen zutiefst ökonomischen Beschränkungen unterworfen ist, ist es wichtig, gemeinsame Standards für alle Intensivbereiche zu formulieren und von fachlicher Seite zu vertreten. Die DIVI-Strukturempfehlungen für Intensivstationen (http://www.divi-org.de/ fileadmin/pdfs/struktur/Empfehlungen_zur_Struktur_von_Intensivstationen_2010-11-30_Kurzversion_ Final.pdf) sind hierfür eine bereits bestehende und geeignete Basis.

Fazit Der strukturierte Einbezug von ausgewählten Angehörigen entspricht dem Interesse des Patienten, ist somit Teil des Behandlungsauftrages und kommt auch der Klinik zu gute. 